\title{
A New Catalytically Active Colloidal Platinum Nanocatalyst: The Multiarmed Nanostar Single Crystal
}

\author{
Mahmoud A. Mahmoud, ${ }^{\dagger}$ Christopher E. Tabor, ${ }^{\dagger}$ Mostafa A. El-Sayed, ${ }^{*, \dagger}$ Yong Ding, ${ }^{\ddagger}$ and \\ Zhong Lin Wang ${ }^{\ddagger}$ \\ Laser Dynamics Laboratory, School of Chemistry and Biochemistry, Georgia Institute of Technology, Atlanta, \\ Georgia 30332-0400 and School of Materials Science and Engineering, Georgia Institute of Technology, \\ Atlanta, Georgia 30332-0245
}

Received December 4, 2007; E-mail: mostafa.el-sayed@chemistry.gatech.edu

\begin{abstract}
Nanocatalysis has attracted great attention in the past two decades, both in heterogeneous solution-phase colloidal reactions ${ }^{1-3}$ and in heterogeneous supported nanoparticle gas-phase reactions. ${ }^{3,4}$ Our group published a Science report early on showing that platinum nanoparticles can be synthesized in a variety of shapes ${ }^{5}$ (tetrahedral, cubic, and truncated octahedral). In that report, it was suggested that catalysis could be shape-dependent, and since that time the field has developed extremely rapidly. ${ }^{6-9}$ Narayanan and El-Sayed ${ }^{6}$ later showed that, indeed, the activation energy of the nanocatalyzed electron-transfer reaction between thiosulfate and hexacyanoferrate III is shape-dependent in colloidal nanocatalysis in which nanocatalysts that have more atoms on edges or corners (i.e., more valency unsaturated atoms) possess more catalytic activity. More recently, shape-dependence was also found to occur in heterogeneous gas-phase nanocatalysis. ${ }^{7,8}$ Furthermore, we found that the catalytically active nanoparticles undergo shape changes to the less active nanospheres, causing them to lose some of their activity. ${ }^{10-12}$ The possible mechanism for shape change and particle growth was recently discussed where the high-resolution TEM images were reported. ${ }^{13}$ Previously, ${ }^{14}$ tetrahedral platinum nanoparticles have been prepared by the hydrogen reduction technique, and different sizes were synthesized by changing the ratio of the preprepared platinum seed concentration to the $\mathrm{Pt}^{+2}$ concentration. Very recently in a Science report, ${ }^{3}$ tetrahexahedral platinum nanocrystals with high index facets, i.e., rich in active valency unsaturated atoms, have been synthesized electrochemically. The authors showed that they are also catalytically very active. These particles were prepared and studied on the surface of an electrode and were not transferable to colloidal solutions.
\end{abstract}

In the present work, tetrahedral platinum nanocrystals (TPNs) were used as seeds for the preparation of a new shape of a colloidal platinum nanostar, without the need for organic solvents, templates, ion replacements, or substrates. The TPNs used were themselves synthesized in large yields by a simple new technique. The lowand high-resolution TEM of the nanostar particles are determined, and the number of arms on each nanostar is found to vary from particle to particle, ranging from a few to over 30 . More interesting is that even the largest nanostars are found to form single crystals. This strongly suggests a mechanism of formation involving seeded growth rather than an aggregation or an assembly process of the seed particles. The catalytic activity for these multiarmed nanostar platinum single crystals is examined for the reaction between hexacyanoferrate III (HCFIII) and thiosulfate and found to be higher than the platinum TPN, which are known to be the most active shape of this metal in colloidal solutions.

To prepare the tetrahedral platinum nanocrystals (TPNs), 0.0667 $\mathrm{g}$ of PVP (MW 360000 ) was dissolved in $33 \mathrm{~mL}$ of deionized water, 4 drops of $1 \mathrm{M} \mathrm{HCl}$ were added, and the solution was heated

† School of Chemistry and Biochemistry.

$\$$ School of Materials Science and Engineering.

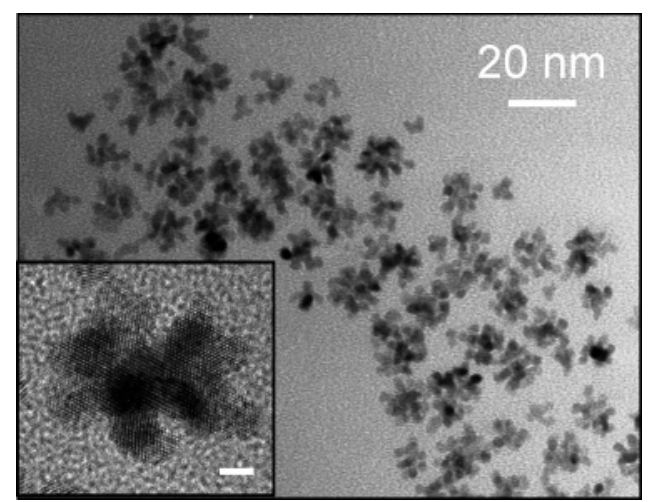

Figure 1. TEM image of new mulitarmed nanostar particles. Inset is HRTEM image of nanostar displaying multiple arms branching in various directions from the initial seed particle. The scale bar in the insert is $2 \mathrm{~nm}$.

to boiling. A $3 \mathrm{~mL}$ aliquot of $0.01 \mathrm{M} \mathrm{K}_{2} \mathrm{PtCl}_{4}$ was added, and the resulting solution was allowed to boil for $2 \mathrm{~min}$. After $2 \mathrm{~min}$ of boiling, $14 \mathrm{~mL}$ of ethanol were rapidly injected into the solution. The solution was left for $10 \mathrm{~min}$ to cool gradually to room temperature, and the solution color changed from colorless to dark black. The solution was left overnight in the dark at $4{ }^{\circ} \mathrm{C}$ before being used in the catalytic reaction. To prepare the nanostar platinum nanoparticles, $0.133 \mathrm{~g}$ of PVP (MW 360000 ) was dissolved in $100 \mathrm{~mL}$ of deionized water. An 8 drop aliquot of $1 \mathrm{M}$ $\mathrm{HCl}$ was added followed by $9.6 \mathrm{~mL}$ of $0.01 \mathrm{M} \mathrm{K}_{2} \mathrm{PtCl}_{6}$, and the resulting solution was brought to boil. A $40 \mathrm{~mL}$ aliquot of the TPN solution, synthesized as described above, was added. The solution was brought to boil, and $36 \mathrm{~mL}$ of ethanol were injected. The resulting solution was refluxed for $3 \mathrm{~h}$. The Pt nanostar nanoparticles were imaged by electron microscopy using a JEOL-4000EX highresolution transmission electron microscope (HRTEM) at $400 \mathrm{kV}$. The reaction was initiated by simultaneously injecting $0.1 \mathrm{~mL}$ of $0.1 \mathrm{M} \mathrm{HCFIII} \mathrm{and} 0.1 \mathrm{~mL}$ of $1.0 \mathrm{M}$ thiosulfate into a $9.8 \mathrm{~mL}$ platinum nanoparticle solution $(\mathrm{pH}=7)$. A Shimadzu UV-visNIR spectrophotometer was used to follow the kinetics of the catalyzed reactions by monitoring the decreasing rate of the HCFIII absorption band at $420 \mathrm{~nm}$. The reaction rate constant was calculated at various temperatures $\left(27,35,45\right.$, and $\left.55^{\circ} \mathrm{C}\right)$ over 5 min intervals. An Arrehnius plot was used to calculate the activation energy of the reaction when using each catalyst.

The shape and size of the new nanoparticles were characterized by HRTEM. Statistical analysis was performed for 300 particles randomly selected from three different preparations. The TPN had a size of $3.9 \pm 2.4 \mathrm{~nm}$ with a shape distribution of $66 \pm 5 \%$ tetrahedral shape, $23 \pm 2 \%$ distorted tetrahedral shape, and $11 \pm$ $2 \%$ spherical shape. The new synthetic method gives high TPN yields. Figure 1 shows HRTEM images of the multiarmed nanostar single-crystal nanoparticles. The particle size was measured as the largest distance between two points on a single particle and was 


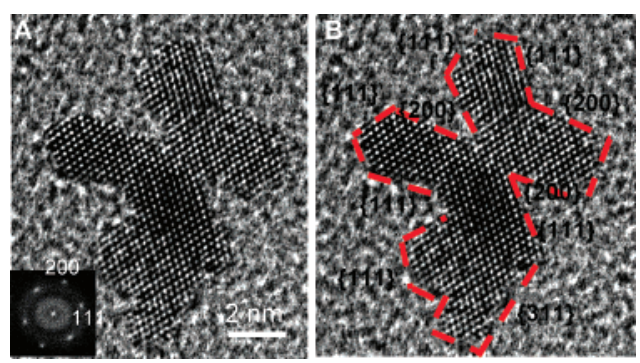

Figure 2. HRTEM image of a multiarmed single-crystal nanostar. The inset in (A) shows the Fast Fourier Transform (FFT) diff. pattern of the nanostar, indicating that the particle is single crystalline. Surface crystal facets are indexed in (B), with some high index $\{311\}$ crystal planes exposed.

found to be $11.2 \pm 2.7 \mathrm{~nm}$ when the size distribution was fit to a Guassian shape (Figure S1). The three-dimensional multiarmed (greater than two arms) star shape constituted $>90 \%$ of the particles in solution. Figure 2 is an HRTEM image of a single multiarmed nanostar. The inserted Fast Fourier Transform (FFT) pattern of the HRTEM image shown in the inset of Figure 2A indicates that the star shaped particle is a single crystal and can be indexed by an incident electron beam along the [011] direction. Most of the exposed surfaces belong to $\{111\}$ and $\{200\}$ planes, which are identified in Figure 2B. At the same time, a small amount of high index planes, such as $\{311\}$, can also be identified.

The single crystalline nature of the nanostar particles can be used to elucidate its mechanism of formation from the initial seed $\mathrm{Pt}$ TPN. This mechanism begins with a single crystalline seed particle, from which the nanostar arms grow as the Pt(IV) salt is reduced. The arms possess the same crystalline structure as the initial seed particle, giving the entire particle a single crystalline structure, which is identified using the FFT pattern in the insert of Figure 2A. The growth direction is dictated by the rate of capping of different facets on the particle. The number of arms on each multiarmed nanostar single-crystal particle is uniquely determined by both the amount of $\mathrm{Pt}(\mathrm{IV})$ salt reduced around the particle and the rate of capping of the particles by the PVP-360 capping agent. The single crystalline nature of the multiarmed nanostars rules out the mechanism that involves the aggregation of multiple seed particles to form a single, larger particle. This would have resulted in the formation of many twinning planes in the structure, which were not observed.

An Arrhenius plot was used to determine the activation energies $\left(E_{\mathrm{a}}\right)$ of the reaction between HCFIII and thiosulfate. The slope of the linear relationship between the natural logarithm of the temperature-dependent rate constant and the inverse of temperature is $-E_{\mathrm{a}} / R$, where $R$ is the universal gas constant. The value of the activation energy $E_{\mathrm{a}}$ is used because it is independent of concentration and the nanocatalyst surface area. The activation energy for the reaction between HCFIII and thiosulfate in the absence of catalyst was measured to be $38.3 \pm 2 \mathrm{~kJ} / \mathrm{mol}$, in agreement with the literature value. ${ }^{15,16}$ The activation energy of the reaction was found to be $23.0 \pm 2.5 \mathrm{~kJ} / \mathrm{mol}$ when the TPN were used (Figure S2A). The nanostar PtNPs were found to give a lower activation energy of $14.1 \pm 1.7 \mathrm{~kJ} / \mathrm{mol}$ (Figure S2B), which is lower than that for the tetrahedral shape having the same capping agent and under the same conditions, indicating that the nanostar shape is a more active nanocatalyst than the tetrahedral one, which itself is known to be a highly active nanocatalyst. ${ }^{6}$ Appropriate control experiments were preformed to ensure that excess Pt(II) and Pt(IV) ions were not responsible for the catalysis by mixing the ionic metallic salts with the two reactants and monitoring the rate of catalysis. No change in the activation energy value from that found for the uncatalyzed reaction was observed.

The activation energy for the reduction of HCFIII by thiosulfate catalyzed by the TPN was found to be $23.0 \pm 2.5 \mathrm{~kJ} / \mathrm{mol}$. This is larger than that previously reported using TPN prepared by hydrogen reduction $(14.0 \pm 0.6 \mathrm{~kJ} / \mathrm{mol}) .{ }^{17}$ This can be explained in two ways. First, it has been reported ${ }^{18}$ by Sharma et al. that an optimum size of $38 \mathrm{~nm}$ exists for platinum nanoparticles, resulting in the highest catalytic activity of the HCFIII reduction reaction by thiosulfate. As the size decreases from $38 \mathrm{~nm}$, the rate constant for the reaction was reported ${ }^{18}$ to decrease. It is noteworthy that the TPNs prepared using the $\mathrm{Pt}(\mathrm{II})$ salt as reported here $(3.9 \mathrm{~nm})$ are smaller in size than the TPNs used by Narayanan and El-Sayed $(5.1 \mathrm{~nm}){ }^{6}{ }^{6}$ This reduction in size will result in our TPNs possessing a slightly lower catalytic activity and, thus, a slightly higher activation energy for the reaction. Second, the percentage of nanoparticles that are tetrahedral in shape was slightly lower using the method described here $(66 \pm 5 \%)$ than previously reported by Narananan and El-Sayed $(76 \pm 3 \%){ }^{6}$ The lower percentage of the highly active TPNs in our synthesis would contribute to the observed higher activation energy value that we report here for TPNs.

In conclusion, we have described a simple method to prepare novel nanoparticle shapes with high yield, which possess a unique single-crystal nanostructure with high catalytic activity: a multiarmed nanostar and a tetrahedral shape. The mechanism of formation of the multiarmed nanostar single crystal is concluded to be a seeded growth mechanism from the TPN. The activation energy for the reduction of ferricyanide by thiosulfate is found to be much smaller when catalyzed by the nanostar $\left(E_{\mathrm{A}}=14 \mathrm{~kJ} / \mathrm{mol}\right)$ than by the seed TPN from which it was made $(23 \mathrm{~kJ} / \mathrm{mol})$. This was explained by the presence of the multiple arms of the nanostar with more edges and corners as well as the presence of high index sites observed in the HRTEM images of the nanostar structure.

Acknowledgment. The authors would like to thank NSF support under Grant No. NSF CHE 0554668. We thank the Georgia Tech Electron Microscopy Center for the HRTEM microscope we used to carry out this work.

Supporting Information Available: Arrhenius plots used to measure the catalytic activation energy. This material is available free of charge via the Internet at http://pubs.acs.org.

\section{References}

(1) Freund, P. L.; Spiro, M. J. Phys. Chem. 1985, 89, 1074-7.

(2) Li, Y.; Hong, X. M.; Collard, D. M.; El-Sayed, M. A. Org. Lett. 2000, 2 , 2385-2388.

(3) Tian, N.; Zhou, Z.-Y.; Sun, S.-G.; Ding, Y.; Wang, Z. L. Science (Washington, D.C.) 2007, 316, 732-735

(4) Strongin, D. R.; Carrazza, J.; Bare, S. R.; Somorjai, G. A. J. Catal. 1987, $103,213-15$.

(5) Ahmadi, T. S.; Wang, Z. L.; Green, T. C.; Henglein, A.; El-Sayed, M. A. Science (Washington, D.C.) 1996, 272, 1924-1926.

(6) Narayanan, R.; El-Sayed, M. A. Nano Lett. 2004, 4, 1343-1348.

(7) Lee, H.; Habas, S. E.; Kweskin, S.; Butcher, D.; Somorjai, G. A.; Yang, P. Angew. Chem., Int. Ed. 2006, 45, 7824-7828.

(8) Rioux, R. M.; Song, H.; Grass, M.; Habas, S.; Niesz, K.; Hoefelmeyer, J. D.; Yang, P.; Somorjai, G. A. Top. Catal. 2006, 39, 167-174

(9) Somorjai, G. A.; Bratlie, K. M.; Montano, M. O.; Park, J. Y. J. Phys. Chem. B 2006, 110, 20014-20022.

(10) Narayanan, R.; El-Sayed, M. A. J. Phys. Chem. B 2003, 107, 1241612424.

(11) Narayanan, R.; El-Sayed, M. A. J. Am. Chem. Soc. 2004, 126, 71947195.

(12) Narayanan, R.; El-Sayed, M. A. J. Phys. Chem. B 2004, 108, 57265733.

(13) Narayanan, R.; El-Sayed, M. A. J. Phys. Chem. B 2005, 109, $12663-$ 12676.

(14) Kinge, S.; Boennemann, H. Appl. Organomet. Chem. 2006, 20, 784787

(15) Li, Y.; Petroski, J.; El-Sayed, M. A. J. Phys. Chem. B 2000, 104, 1095610959 .

(16) Song, Y.; Yang, Y.; Medforth, C. J.; Pereira, E.; Singh, A. K. Xu, H Jiang, Y.; Brinker, C. J.; van Swol, F.; Shelnutt, J. A. J. Am. Chem. Soc 2004, 126, 635-645.

(17) Narayanan, R.; El-Sayed, M. A. J. Am. Chem. Soc. 2003, 125, 83408347.

(18) Sharma, R. K.; Sharma, P.; Maitra, A. J. Colloid Interface Sci. 2003, $265,134-140$

JA710646T 